\title{
Observation of a Dissipative Phase Transition in a One-Dimensional Circuit QED Lattice
}

\author{
Mattias Fitzpatrick, ${ }^{1}$ Neereja M. Sundaresan, ${ }^{1}$ Andy C. Y. Li, ${ }^{2}$ Jens Koch, ${ }^{2}$ and Andrew A. Houck ${ }^{1}$ \\ ${ }^{1}$ Department of Electrical Engineering, Princeton University, Princeton, New Jersey 08540, USA \\ ${ }^{2}$ Department of Physics and Astronomy, Northwestern University, Evanston, Illinois 60208, USA \\ (Received 10 August 2016; revised manuscript received 19 January 2017; published 10 February 2017) \\ Condensed matter physics has been driven forward by significant experimental and theoretical progress \\ in the study and understanding of equilibrium phase transitions based on symmetry and topology. However, \\ nonequilibrium phase transitions have remained a challenge, in part due to their complexity in theoretical \\ descriptions and the additional experimental difficulties in systematically controlling systems out of \\ equilibrium. Here, we study a one-dimensional chain of 72 microwave cavities, each coupled to a \\ superconducting qubit, and coherently drive the system into a nonequilibrium steady state. We find \\ experimental evidence for a dissipative phase transition in the system in which the steady state changes \\ dramatically as the mean photon number is increased. Near the boundary between the two observed phases, \\ the system demonstrates bistability, with characteristic switching times as long as $60 \mathrm{~ms}$ - far longer than \\ any of the intrinsic rates known for the system. This experiment demonstrates the power of circuit QED \\ systems for studying nonequilibrium condensed matter physics and paves the way for future experiments \\ exploring nonequilbrium physics with many-body quantum optics.
}

DOI: 10.1103/PhysRevX.7.011016

Over the past decades, there has been remarkable progress in studying both real and synthetic quantum materials. Advances in nanoscale fabrication and cryogenics, for instance, have allowed for exquisite control of electronic systems - unlocking strongly correlated electronic states and topological materials [1]. Simultaneously, the ability to model desired Hamiltonians with ultracold Fermi and Bose gases has allowed unprecedented access to synthetic material properties [2]. As a whole, much of the development of condensed matter physics has focused on the study of (quasi)equilibrium physics, which is more accessible both experimentally and theoretically. However, the constant presence of dissipation, noise, and decoherence belie the fact that, ultimately, the world is nonequilibrium.

A phase transition indicates a sometimes sudden change in the physical properties of a system as a function of some external system parameter. Thermal phase transitions are well understood in the context of statistical mechanics and occur when the free energy of the system becomes nonanalytic. At zero temperature, the role of quantum fluctuations gives rise to a new set of quantum phase transitions that involve a sudden change in the ground state of a Hamiltonian; a phase transition occurs when the gap between the first excited state and the ground state closes. These concepts need to be extended to consider nonequilibrium steady

Published by the American Physical Society under the terms of the Creative Commons Attribution 3.0 License. Further distribution of this work must maintain attribution to the author(s) and the published article's title, journal citation, and DOI.
Subject Areas: Condensed Matter Physics,

Photonics, Quantum Physics states, as the system is no longer in its ground state but rather in a state that balances drive and dissipation. In a dissipative phase transition, the steady state abruptly changes as a system parameter is varied [3]. Whenever the system is describable in terms of a Lindblad master equation [4], $\dot{\rho}=\mathbb{L} \rho$, where $\rho$ is the density matrix, such a transition is signaled by the closing of the lowest excitation gap in the spectrum of the Liouvillian superoperator $\mathbb{L}$.

In recent years, interacting photons have emerged as an excellent candidate for studying nonequilibrium condensed matter physics due to the lack of particle number conservation $[5,6]$. In cavity quantum electrodynamics, strong coupling between atoms and a cavity can mediate effective photon-photon interactions [7-9]. Arrays of coupled microwave [10] or optical [11] cavities can be fabricated by conventional lithographic techniques, and the competition between on-site interactions and hopping between neighboring cavities can give rise to quantum phase transitions of light [12-14]. A wide range of many-body effects have been predicted in these systems, including a Mott insulatorsuperfluid phase transition [12-14] and fractional quantum Hall-like states of light [15-17]. Experiments on small systems have demonstrated low-disorder lattices [10], a dynamical quantum phase transition in a cavity dimer [18], and chiral ground state currents in a cavity trimer [19]. Circuit quantum electrodynamics (cQED) lattices are inherently open systems, with dissipation an ever-present force that leads to both qubit relaxation as well as the inevitable loss of photons from microwave cavities. While dissipation presents an obstacle for quantum information processing, it is of fundamental interest in the study of 

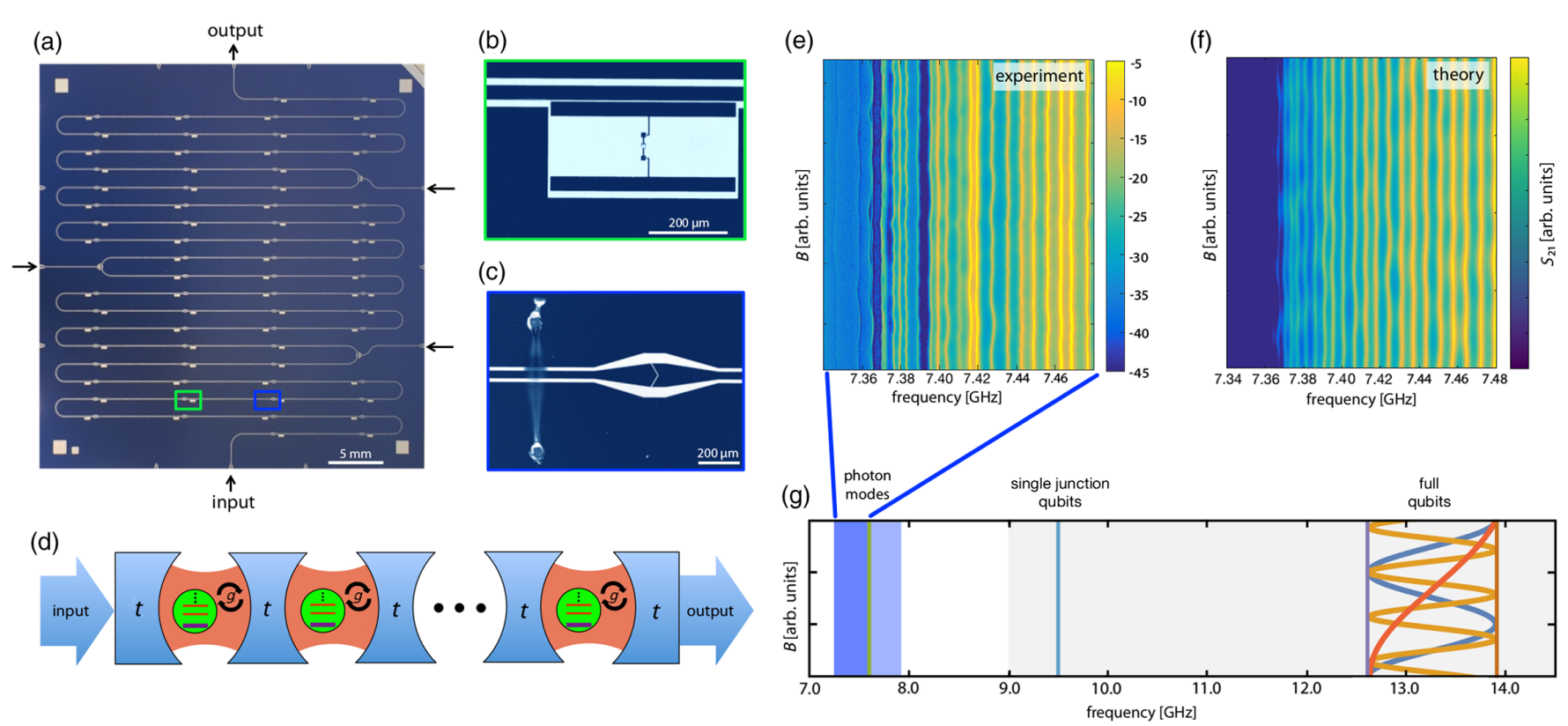

FIG. 1. 72-site circuit QED lattice. (a) Coplanar waveguide resonators, each with a bare cavity frequency $\omega_{c} / 2 \pi=7.6(2) \mathrm{GHz}$, are capacitively coupled to form a linear chain on a $35 \times 35 \mathrm{~mm}^{2}$ chip. Each resonator is coupled to its neighboring resonators with a hopping rate $t / 2 \pi=80(10) \mathrm{MHz}$ and each low-power eigenmode as a measured average photon loss rate of $\kappa / 2 \pi=1.6(5) \mathrm{MHz}$. At three intermediate chain sites, three-way coupling capacitors provide ports for secondary input and output lines (arrows on sides), which allow further characterization of the device. (b),(c) A transmon qubit is capacitively coupled to the center pin near the edge of each resonator in the lattice, ensuring strong coupling to the fundamental mode of each resonator. The coupled resonator-qubit system forms the fundamental unit cell of the lattice. (d) The circuit can be modeled as a linear chain of coupled oscillators, each dispersively coupled to a weakly anharmonic multilevel system. Transmission $\left(S_{21}\right)$ as a function of external magnetic field $(B)$ as measured experimentally (e) and predicted theoretically (f). Numerical simulations reveal qualitative agreement between theory and experiment for experimentally relevant parameters, $\omega_{c} / 2 \pi=7.6 \mathrm{GHz}$, tunable qubit frequency, $\Omega / 2 \pi \in[12.7,13.9]$ GHz, single-junction qubit frequency, $\Omega_{\mathrm{SJ}} / 2 \pi=9.5 \mathrm{GHz}, g / 2 \pi=410 \mathrm{MHz}$ (on resonance with $\omega_{c}$ ), transmon charging energy, $E_{c} / h=350 \mathrm{MHz}$, and $t / 2 \pi=90 \mathrm{MHz}$. By changing the strength of the external magnetic field, the tunable qubit frequencies are continuously changed and produce nonperiodic dispersive shifts due to the random areas of the transmon SQUID loops. (g) The blue rectangles indicate the frequency range of the photonic modes, with the dark blue rectangle indicating the range of frequencies shown in (e) and (f) and the green line indicating the bare cavity frequency. The gray shaded region reflects the predicted uncertainty range of the qubit frequencies (including potential single-junction qubits) and curves represent the qubit frequencies as a function of external magnetic field.

nonequilibrium phase transitions. Just as excitations inevitably leak from the system, it is easy to add photons back and to drive the system into a steady state, making these systems particularly amenable to the study of dissipative phase transitions.

In this paper, we present experimental evidence for a dissipative phase transition in a CQED lattice. We observe that at drive frequencies between the low-power resonance frequencies of the system, there exists a region of hysteresis and bistability where the steady state of the system switches stochastically between two states $\rho_{1}$ and $\rho_{2}$. By determining the corresponding switching rates, we can obtain the socalled asymptotic decay rate, which characterizes the closing of the spectral gap of the Liouvillian $\mathbb{L}$. At the transition between the two states, the characteristic switching times become exceptionally long, a key characteristic of a dissipative phase transition. A similar observation has recently been made in a single-cavity system with multiple qubits [20].
Our device, shown in Fig. 1(a), consists of a linear chain of 72 lattice sites. Each site comprises a coplanarwaveguide resonator with fundamental-mode frequency $\omega_{c} / 2 \pi=7.6(2) \mathrm{GHz}$, coupled to a transmon qubit [21] [Fig. 1(b)] placed at one of the resonator's voltage antinodes, and each site is coupled to neighboring sites with a hopping rate $t / 2 \pi=80(10) \mathrm{MHz}$. Variations in transmon qubit frequencies in fabrication are a likely source of uncontrolled disorder that is difficult to compensate for in our lattice, because tuning of individual qubits is infeasible. Instead, we randomize qubit frequencies intentionally to ensure that features of interest are universal to the system rather than artifacts of a particular instance of disorder. To do this, each qubit is fabricated with a SQUID loop of random area, meaning that as a global magnetic field is tuned, the qubit frequencies can be randomized. By using an asymmetric SQUID design [22], we can limit qubit frequencies to a finite range and avoid degeneracies with the resonator array [see Fig. 1(g)]. Because of 
fabrication challenges, roughly $20 \%$ of the qubits have only a single junction (see Supplemental Material, Sec. I, for details [23]). We observe qualitative agreement between measured [Fig. 1(e)] and simulated [Fig. 1(f)] low-power transmission as a function of external magnetic field $B$ for parameters specified in the caption of Fig. 1. A graphical representation of the relevant system frequencies as a function of magnetic field is shown in Fig. 1(g).

To experimentally study the nonequilibrium behavior of the device, we monitor the transmission $\left(S_{21}\right)$ across the lattice while varying the drive frequency and scanning the drive power over more than 5 orders of magnitude [Fig. 2(a)]. At low drive powers, we find the expected discrete transmission peaks associated with the interactionshifted eigenmode frequencies of the resonator lattice. As we vary the mean photon number in the system by increasing the strength of the drive, we observe that a sudden change in system behavior occurs: transmission peaks split and then, at around $-95 \mathrm{dBm}$ of drive power, abruptly give way to a region of strongly suppressed transmission. In this high-power region, peaklike features are completely absent.

The transition between the low- and high-power phases can be more thoroughly explored by measuring the transmission at a single drive frequency while sweeping the drive power either from low to high [Fig. 2(c)] or from high to low [Fig. 2(d)]. Doing so reveals a significant region exhibiting hysteresis, which is located at the top of the low-power lobes where the transition to the high-power phase occurs. Subtracting the transmission signals for the two different sweep directions clearly marks the hysteretic regime, as shown in Fig. 2(e).

To gain insight into this behavior, we model the system as a one-dimensional chain of identical cQED elements, as illustrated in Fig. 1(d). The corresponding Hamiltonian,

$$
H=\sum_{j}\left(H_{j}^{r}+H_{j}^{q}+H_{j}^{\mathrm{rq}}\right)+\sum_{\left\langle j, j^{\prime}\right\rangle} H_{j, j^{\prime}}^{\mathrm{hop}}+H^{d},
$$

includes terms for the resonator $H_{j}^{r}$, qubit $H_{j}^{q}$, and the resonator-qubit coupling on each site $j, H_{j}^{\mathrm{rq}}$, as well as hopping of photons between nearest-neighbor resonators $H_{j, j^{\prime}}^{\text {hop }}$, and a coherent drive (acting only on site 1) $H^{d}$. Each resonator contributes a single harmonic mode, $H_{j}^{r}=\hbar \omega_{c} a_{j}^{\dagger} a_{j}$, where $a_{j}^{\dagger}$ and $a_{j}$ are the creation and annihilation operators for photons on site $j$ and $\omega_{c}$ is the fundamental frequency of the resonator. The low-lying transmon levels are described as an anharmonic oscillator $H_{j}^{q}=\mathrm{P}_{N}\left(\hbar \Omega_{j} b_{j}^{\dagger} b_{j}+\frac{1}{2} U b_{j}^{\dagger} b_{j}^{\dagger} b_{j} b_{j}\right) \mathrm{P}_{N}$, with negative Hubbard or Kerr interaction $U=-E_{c}$. The projectors $\mathrm{P}_{N}$ truncate the Hilbert space to levels $N \lesssim \sqrt{E_{J, j} / 2 E_{c}}$ within the transmons' cosine wells, and the operators $b_{j}^{\dagger}, b_{j}$ create and annihilate qubit excitations. $E_{c}$ and $E_{J, j}$ are the single-electron charging energy and the effective Josephson energies of the transmon qubit. In our model, we include the frequency difference between single-junction (a)

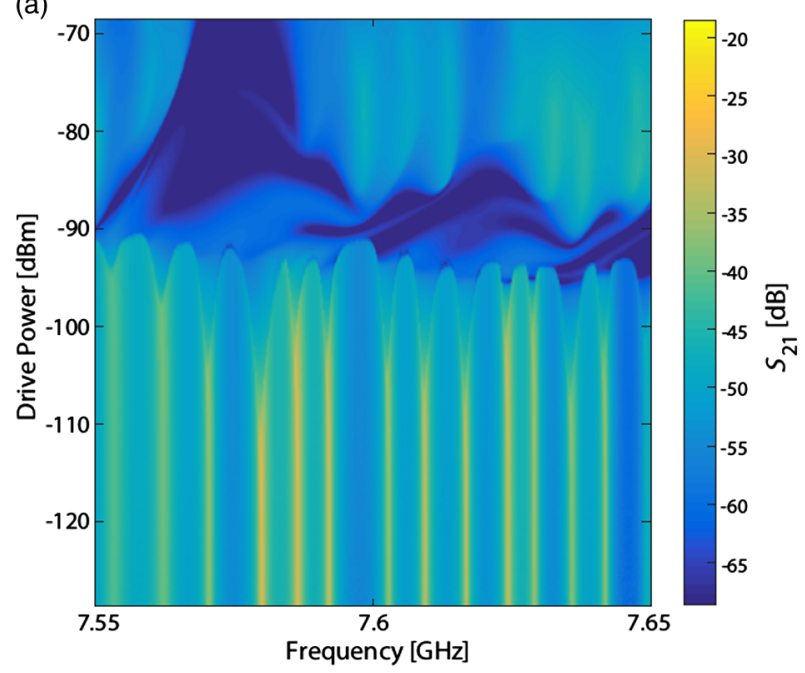

(b)

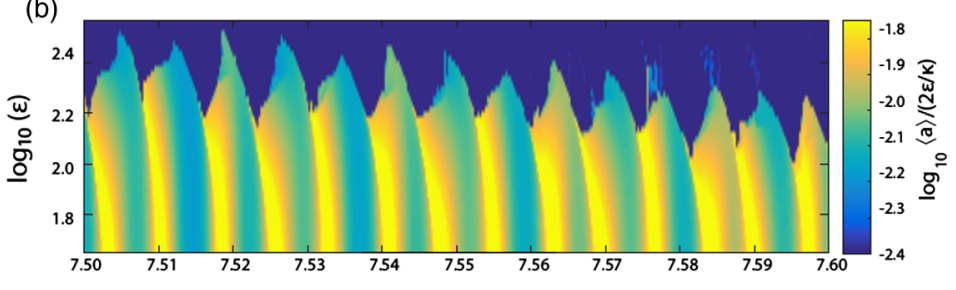

(d)

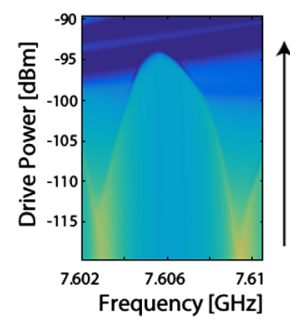

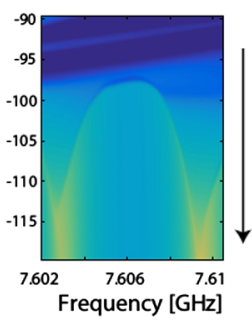

(e)

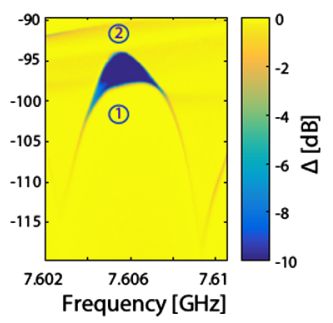

FIG. 2. Microwave transmission $\left(S_{21}\right)$ as a function of power, exhibiting an abrupt transition to a suppressed transmission regime and a region of bistability. (a) Dispersively shifted transmission peaks show nonlinear splitting at increased power and give rise to a region of strongly suppressed transmission without resonance peaks. Data are acquired using frequency sweeps at constant power. (b) Corresponding mean-field results for transmission through a 72-site lattice, showing features qualitatively consistent with the experiment, where $\epsilon$ is the drive power and $\kappa$ is the dissipation rate (qubit and cavity) (c) Zoom into one lobe of the experimental data, showing the sharp transition to a state of suppressed transmission as the drive power is swept from low to high power using constantfrequency, linear power sweep over a 31.95-ms period. (d) Same region as in (c), but sweeping power in the opposite direction (high to low) over the same time period as (c). The transition now occurs at lower power. (e) The difference $\Delta$ in the data shown in (c) and (d) uncovers the large region of hysteresis in between two distinct states (1) and (2). 
and tunable qubits, but neglect disorder otherwise. Qubitresonator coupling and photon hopping take the simple forms $H_{j}^{\mathrm{rq}}=\hbar g_{j}\left(a_{j} b_{j}^{\dagger}+\right.$ H.c. $)$ and $H_{j, j^{\prime}}^{\mathrm{hop}}=\hbar t\left(a_{j} a_{j^{\prime}}^{\dagger}+\right.$ H.c. $)$, where $g_{j}$ and $t$ are the resonator-qubit coupling rate and photon hopping rate, respectively. Within rotating-wave approximation, the microwave drive acting on site 1 is given by $H^{d}=\hbar \epsilon(t) a_{1} e^{i \omega_{d} t}+$ H.c., where $\epsilon$ is the drive strength and $\omega_{d}$ is the drive frequency. In our model, we account for qubit relaxation and intrinsic photon loss (at rates $\Gamma$ and $\kappa$, respectively) by employing the standard Lindblad master equation formalism for the reduced density matrix,

$$
\dot{\rho}=-\frac{i}{\hbar}[H, \rho]+\kappa \sum_{j} \mathbb{D}\left[a_{j}\right] \rho+\Gamma \sum_{j} \mathbb{D}\left[b_{j}\right] \rho,
$$

where $\mathbb{D}[L] \rho=L \rho L^{\dagger}-\frac{1}{2}\left\{L^{\dagger} L, \rho\right\}$ is the usual action of the Lindblad damping operator.

The experimentally observed transition is qualitatively captured by simple, quasiclassical mean-field theory that decouples the sites, but allows for mean-field parameters to differ from site to site. Allowing for site-dependent parameters is particularly relevant for our case, in which the drive acts on only one end of the resonator chain, rather than on every site. Within the quasiclassical treatment [24], the quadrature amplitudes $\alpha_{j}=\left\langle a_{j}\right\rangle$ and $\beta_{j}=\left\langle b_{j}\right\rangle$ play the role of mean-field parameters and obey the system of 144 nonlinear coupled equations:

$i \dot{\alpha}_{j}=\left(\omega_{c}-\omega_{p}-i \frac{\kappa}{2}\right) \alpha_{j}+g_{j} \beta_{j}+t\left(\alpha_{j-1}+\alpha_{j+1}\right)+\epsilon \delta_{j, 1}$,

$i \dot{\beta}_{j}=\left(\Omega_{j}-\omega_{p}-i \frac{\Gamma}{2}\right) \beta_{j}+\frac{U}{\hbar}\left|\beta_{j}\right|^{2} \beta_{j}+g_{j} \alpha_{j}$.

From these equations, we obtain the steady-state transmission signal $S_{21} \sim\left\langle a_{j}\right\rangle$ and the second-order coherence function $g^{(2)}(0)=\left\langle a_{j}^{\dagger} a_{j}^{\dagger} a_{j} a_{j}\right\rangle /\left|\left\langle a_{j}^{\dagger} a_{j}\right\rangle\right|^{2}$, choosing $j$ as the label of the output port resonator (see the Methods section).

The mean-field steady-state transmission [Fig. 2(b)] reproduces the qualitative features of the experimental data. We find that higher transmon levels are a crucial ingredient to the model, as calculations based on the simpler Jaynes-Cummings lattice do not yield results consistent with experiment. Figure $1(\mathrm{~g})$ indicates that the lowest frequency transition of the transmons $\Omega_{0 \rightarrow 1}$ is well above the photonic modes of the system. However, the transmon energy level structure is known to have a negative anharmonicity [25], which implies that the transition frequencies between adjacent transmon levels become smaller for higher levels. From numerical simulations, we find that when the transmon level $n$ is predicted to have large occupation in the high-power state, the transition frequency between $n$ and $n+1, \Omega_{n \rightarrow n+1}$, is (quasi)resonant with the set of photonic modes. This means that after crossing into the high-power state, photons in the lattice can resonantly exchange excitations with the transmonsintroducing strong effective photon-photon interactions. Interactions of this type are likely to explain the scattering observed in the power spectrum we show below (and see Fig. 6 in the Supplemental Material [23]). In addition, the drop in transmission in the high-power phase exhibits chaotic dynamics which resembles results previously obtained for a driven, dissipative Bose-Hubbard chain [24].

It is important to note that bistability has recently been predicting in other driven-dissipative photonic lattices [26], smaller nonlinear systems such as single-site circuit QED systems [27], and Duffing oscillators [28]. For the latter system, nonequilibrium physics and bistability can be rigorously analyzed by studying minima of the quasienergy potential in a rotating frame, as well as the processes leading to switching between minima $[28,29]$. This type of analysis, however, becomes very challenging once multiple nonlinear oscillators are coupled together. The low operating temperature of the device, as well as definite deviations between experimental data and the quasiclassical meanfield treatment, may indicate the importance of quantum effects. Our observed switching rates, shown in Fig. 3, are many orders of magnitude slower than switching rates of other single-site bistable cQED devices, such as Josephson bifurcation amplifiers [27], pointing to the suppression of switching due to collective effects in our CQED chain. Bistability and hysteresis can further be produced dynamically in a Bose-Hubbard dimer, as discussed by Drummond and Walls [30] and recently by Casteels et al. [31]. As shown in Ref. [31], hysteresis arises from parameter sweeps across a point where the spectral gap of the Liouvillian superoperator $\mathbb{L}$ (nearly) closes, in a manner analogous to the Kibble-Zurek mechanism [32]. Similar to the case studied by Casteels et al., we find that mean-field theory can capture certain qualitative aspects of the bistability and hysteresis, but cannot capture the dependence of switching rates on sweep times or effects from quantum fluctuations.

The dramatic suppression of transmission and loss of all resonance peaks beyond a certain drive power threshold are indicative of a dissipative phase transition, arising from the intricate interplay of dissipation, driving, and nonlinearity of the system. The crucial quantity for such a transition is the gap in the spectrum of the Lindblad superoperator $\mathbb{L}$. Once the real part of one of its eigenvalues approaches zero, deviations of the steady state along the "direction" of the corresponding $\mathbb{L}$ eigenstate become increasingly long-lived and ultimately allow for a new steady state to emerge. The negative real part of the eigenvalue $\lambda$ closest to zero, $-\operatorname{Re} \lambda$, is known as the asymptotic decay rate (ADR) [3]. An approximation for the ADR can be extracted by single-shot measurements of the dynamics in the bistable region as follows. 

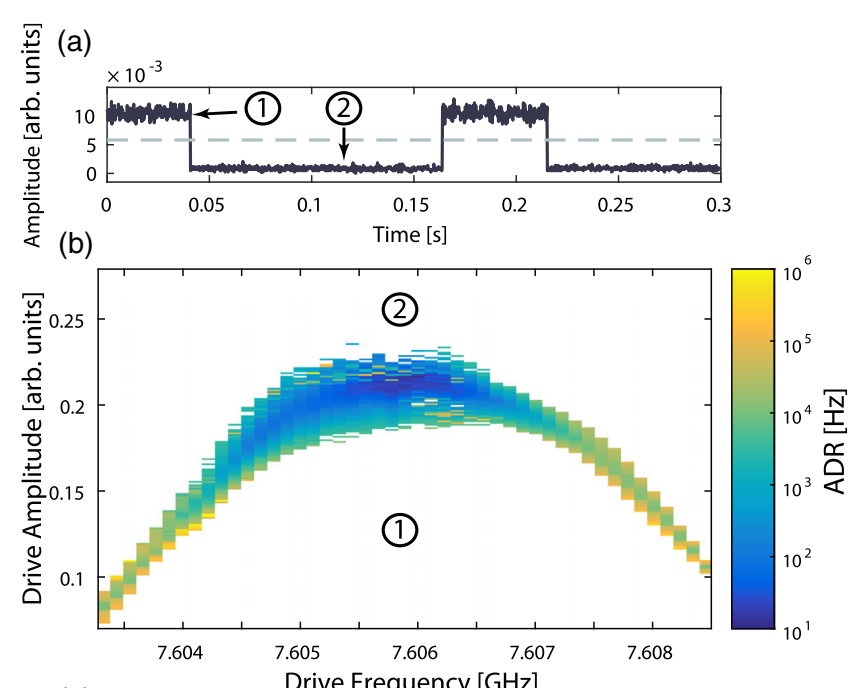

(c)

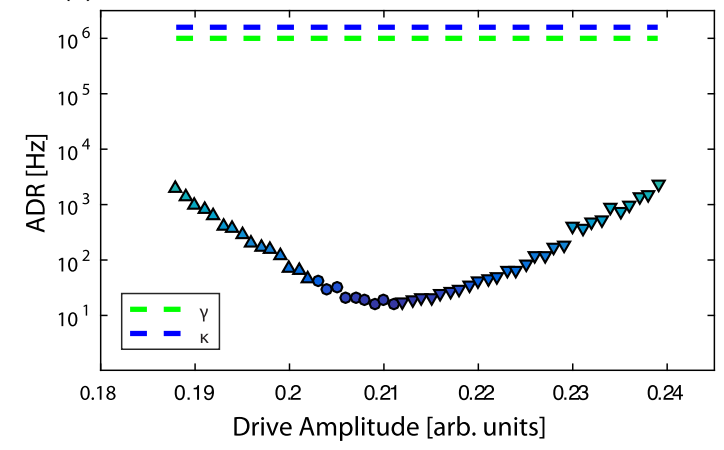

FIG. 3. Asymptotic decay rate in the transition region. (a) Single-shot time trace of the homodyne phase in the hysteretic region for constant drive amplitude. Data show stochastic switching between two distinct metastable states (1) and (2) on time scales vastly exceeding those intrinsic to the system. (b) Asymptotic decay rate obtained from the sum of the characteristic switching times $\gamma_{1 \rightarrow 2}+\gamma_{2 \rightarrow 1}$ as a function of drive frequency and power. (c) ADR for a drive frequency of $7.6059 \mathrm{GHz}$ is plotted as a function of power. $\kappa$ and $\gamma$ are included for reference to indicate that ADR can be as large as 5 orders of magnitude slower than relevant time scales of the device. When either $\gamma_{1 \rightarrow 2}$ or $\gamma_{2 \rightarrow 1}$ are slower than the duration of the measurement pulse $\tau_{m}$, we cannot reliably extract a characteristic switch rate. In these cases, we select the smallest extracted switching time, which is larger than $1 / \tau_{m}$. Upward- (downward-) pointing triangles indicate when $\gamma_{1 \rightarrow 2}\left(\gamma_{2 \rightarrow 1}\right)$ are less than $1 / \tau_{m}$, circles indicate when both rates are used.

In the region of bistability, we apply a drive with constant frequency and amplitude, and record single-shot time traces of the homodyne amplitude and phase. Our measurements show that the system undergoes switching between two metastable states on time scales large compared to systemintrinsic time scales $(\tau \sim \mu \mathrm{s})$ [Fig. 3(a)]. The state of the system at each point along a single-shot trajectory is classified as either $\rho_{1}$ or $\rho_{2}$, and characteristic dwell times are extracted. The statistics acquired from many single-shot trajectories allow us to extract average rates $\gamma_{1 \rightarrow 2}, \gamma_{2 \rightarrow 1}$ for the switching between the two metastable states $\rho_{1}$ and $\rho_{2}$ observed at low power and high power, respectively (labeled as (1) and (2) in the figure).

The extracted switching rates allow us to estimate the asymptotic decay rate by adopting a simplified rateequation model [33] describing the probabilities $p_{1}$ and $p_{2}$ for the system to be in metastable state $\rho_{1}$ or $\rho_{2}$ (see the Methods section for details):

$$
\frac{d}{d t}\left(\begin{array}{l}
p_{1} \\
p_{2}
\end{array}\right)=\left(\begin{array}{cc}
-\gamma_{1 \rightarrow 2} & \gamma_{2 \rightarrow 1} \\
\gamma_{1 \rightarrow 2} & -\gamma_{2 \rightarrow 1}
\end{array}\right)\left(\begin{array}{l}
p_{1} \\
p_{2}
\end{array}\right) .
$$

Diagonalization of this system yields the stationary eigenmode $\rho_{s}=\left(\gamma_{2 \rightarrow 1} \rho_{1}+\gamma_{1 \rightarrow 2} \rho_{2}\right) / \gamma_{\Sigma}$ and the purely decaying eigenmode $\rho_{\mathrm{ADR}}=\gamma_{2 \rightarrow 1} \rho_{1}-\gamma_{1 \rightarrow 2} \rho_{2}$ with corresponding eigenvalues zero and $\lambda_{\mathrm{ADR}}=-\gamma_{\Sigma}=-\left(\gamma_{1 \rightarrow 2}+\gamma_{2 \rightarrow 1}\right)$. Hence, this simplified model predicts an asymptotic decay rate of $-\operatorname{Re} \lambda_{\mathrm{ADR}}=\gamma_{\Sigma}$.

Remarkably, the asymptotic decay rate, shown in Figs. 3(b) and 3(c), reaches a minimum value as low as $\sim 10 \mathrm{~Hz}$, which is 5 orders of magnitude lower than the rates set by photon decay and transmon relaxation in our system. This vast time scale discrepancy delivers strong evidence for the onset of a dissipative phase transition. Similar to the situation of equilibrium phase transitions, it is only in the thermodynamic limit that the spectral gap can fully close and turn the crossover between two steadystate phases into a phase transition in the strict sense [34].

We gather additional evidence for the approach to a dissipative phase transition by measuring fluorescence power spectra and second-order coherence functions in our system. To this end, two different driving pulse shapes, Figs. 4(a) and 4(b), are used to access the distinct states of the system. Within the region of bistability, we can perform state initialization either in the low-power phase (1) or the high-power phase (2) by approaching the final drive amplitude $\xi$ either from a lower or a higher drive amplitude, respectively. After this initialization period, the two pulses maintain the constant drive amplitude $\xi$, during which time the transmitted signal is detected using heterodyne detection with a 32-MHz intermediate frequency. The power spectrum is then obtained by performing a Fourier transform on the heterodyne signal. The second-order correlation function $g^{(2)}(0)$ is measured using techniques outlined in Ref. [35]. Figure 4 indicates that the low-power state can be characterized by a single, coherent drive tone $\left[g^{(2)}(0)=1\right]$ and that the high-power state can be characterized by broadband and multimode (see Supplemental Material, Sec. IV [23]) emission and bunching $\left[g^{(2)}(0) \approx 2\right]$. In addition, the onset of the high-power state has a stark linewidth broadening of the drive tone and a region of strong bunching $g^{(2)}(0) \approx 5$ for the down pulse as the system transitions to the high-power state. Mean-field results for $g^{(2)}$ are in reasonable agreement with the experimental 
measurement data of $g^{(2)}$ in the region below and well above the regime of bistability [Figs. 4(e) and 4(f)]. However, mean-field theory fails to predict experimental results within the region of bistability itself, thus pointing to the possible relevance of quantum effects beyond the quasiclassical
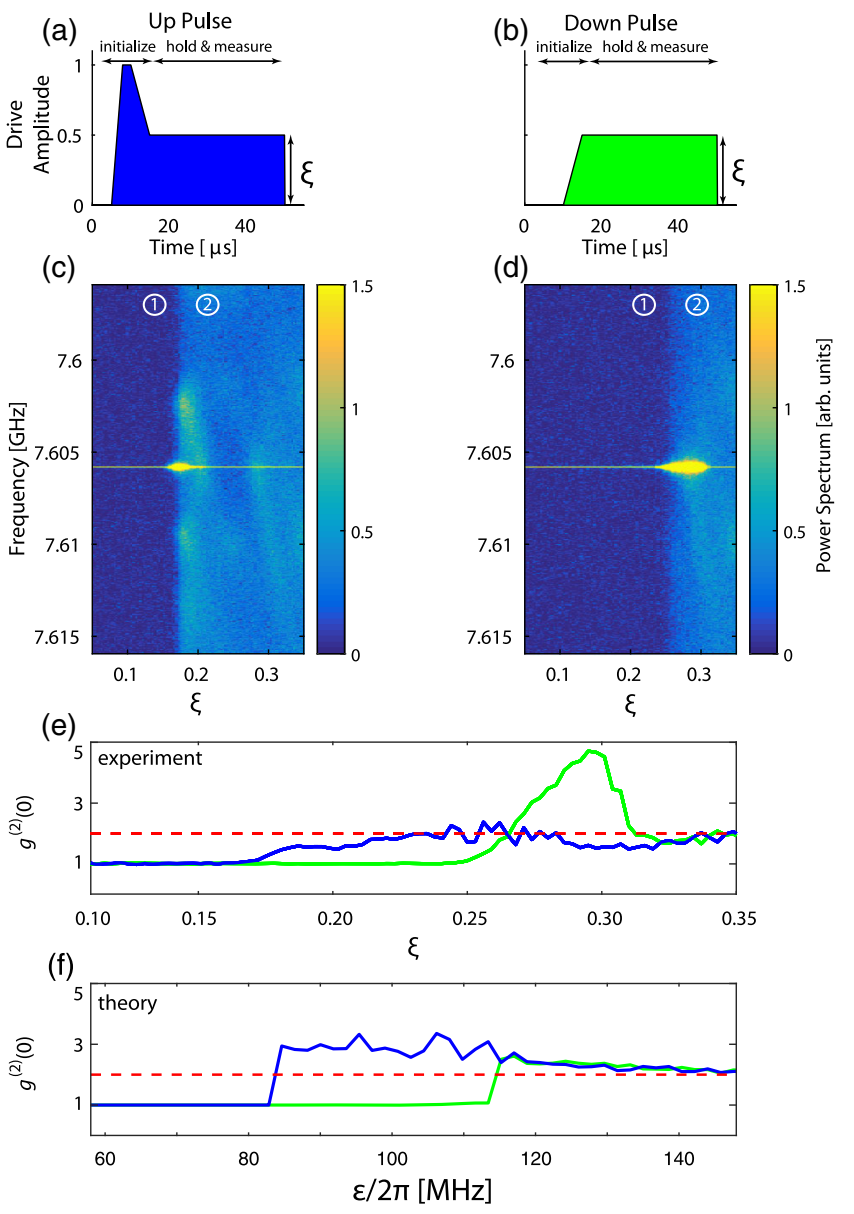

FIG. 4. State characterization. To probe properties of the states within the region of bistability, two pulse sequences are used to initialize the system: (a) an "up pulse" for initialization in the high-power state and (b) a "down pulse" for initialization in the low-power state. Both (a) and (b) return to a constant amplitude $\xi$. In the region of bistability where the ADR is small, the system can be deterministically initialized in (1) or (2) or 193 after which, during the "hold and measure" segment of the pulse sequence, properties of the emitted light can be extracted. The power spectrum in (c) and (d) exhibits that (1) and (2) have drastically different emission properties and that the two states are maintained by a constant, coherent tone, even in the region of bistability [where (c) and (d) show different emission]. Similarly, the second-order correlation function $g^{(2)}(0)$ in (e) can be obtained for each state independently. Results in (c)-(e) indicate that (1) can be characterized by a single, coherent $\left[g^{(2)}(0)=1\right]$ tone at the drive frequency, $7.606 \mathrm{GHz}$, and that (2) can be characterized by broadband emission and bunching $\left[g^{(2)}(0)>1\right]$ at the drive frequency. (f) Mean-field results for the second-order correlation function for comparison. Note that the numerical values on the $x$ axes cannot be compared directly for (e) and (f). description. Based on our modeling, the experiment involves both large numbers of photons and excitations of higher transmon levels, and, hence, may indeed approach the thermodynamic limit necessary for the observation of a dissipative phase transition.

This work demonstrates the exciting capacity of circuit QED lattices to act as a controllable platform guiding deeper theoretical and experimental understanding of nonequilibrium condensed matter physics. A number of interesting experiments are on the immediate horizon, and even more are easily envisioned as long-term objectives. For instance, Hamiltonian tomography of medium-size lattices [36] can be used to gather detailed information on resonator frequencies and hopping elements. Once combined with active tuning of individual qubits, disorder can be eliminated or added in a systematic manner. In this way, qubits can further be brought into exact resonance with cavities or cavity modes, thus increasing the strength of interactions and lowering the power at which phase transitions are anticipated to occur. With the utilization of readily available parametric amplifiers, correlation-function measurements can be performed systematically and harnessed to investigate the importance of quantum effects. Two-point correlators across the lattice could probe long-range coherence or entanglement. Furthermore, a recent paper suggests that our system also undergoes a dissipative phase transition when tuning the photon hopping strength [26], which can be tuned with SQUID-based resonator couplers [37].

In the longer term, this platform can also be harnessed for preparation and stabilization of exotic many-body states of photons. One outstanding challenge of interest is preparation and stabilization of interesting many-body states. In these photonics lattices, the equilibrium phase diagram is rarely of interest as the ground states corresponds to the vacuum state unless we are approaching the ultrastrong coupling limit of circuit QED. In this work, we begin to investigate the nonequilibrium steady state of the system, and it would be interesting to make connections between this approach and the ground state of an equilibrium system. One can also stabilize interesting many-body states with clever reservoir engineering [38,39] or the introduction of an artificially generated chemical potential [40]. Finally, among the host of theory proposals, a particularly exciting future direction is that of breaking time-reversal symmetry in photonic lattices and realizing fractional quantum Hall states of bosons [15-17].

Methods: Experimental methods.-The cavities of the circuit QED lattice are etched using standard optical lithography and plasma etching techniques from a 200nm-thick $\mathrm{Nb}$ film on a $500-\mu \mathrm{m}$-thick, $35 \times 35 \mathrm{~mm}^{2}$ sapphire substrate. Transmon qubits are designed to have Josephson junctions with dimensions $200 \times 180 \mathrm{~nm}^{2}$ and $450 \times 450 \mathrm{~nm}^{2}$ and are fabricated according to the "Manhattan" technique outlined by Potts et al. [41], using electron beam lithography and aluminum evaporation. 
Similar transmon qubits have coherence times $T_{1}=1 \mu \mathrm{s}$, a coupling constant of $g / 2 \pi=450 \mathrm{MHz}$ (on resonance with $\omega_{c}$ ), and charging energy $E_{c} / h=350 \mathrm{MHz}$.

Measurements are performed at a temperature of $7.5 \mathrm{mK}$ in a dilution refrigerator, and inside a superconducting solenoid magnet controlled by a room-temperature dc voltage source. Transmission measurements are performed using a network analyzer, switching-rate measurements using standard homodyne detection techniques. All powerspectrum measurements are done by taking the Fourier transform of a heterodyne signal, and $g^{(2)}$ measurements are implemented using the homodyne techniques described by Eichler et al. [35].

Numerical solution of the mean-field equations.-We solve for the stationary state of the mean-field equations (3) by time evolution and extracting the long-time limit, since root-finding methods are difficult to handle for the large system of nonlinear equations [24]. In the high-power phase the dynamics is chaotic, so that additional time averaging in the long-time limit is required. For instance, the second-order coherence function is obtained by evaluating $g^{(2)}(0)=\left\langle\left\langle|\alpha(t)|^{2}\right\rangle\right\rangle_{t} / \mid\left\langle\left.\langle|\alpha(t)|\rangle_{t}\right|^{2}\right.$, where the time average $\left\langle\langle\cdot\rangle_{t}\right.$ is carried out over a time interval that excludes any initial transient behavior.

Model underlying the ADR estimate.-First, consider stochastic switching between two pure states $|1\rangle$ and $|2\rangle$. The simplest description is based on a two-level Hamiltonian $H=E_{21}|2\rangle\langle 2|$, where $E_{21}$ is the energy difference between the two states, and the master equation

$\dot{\rho}=-\frac{i}{\hbar}[H, \rho]+\gamma_{1 \rightarrow 2} \mathbb{D}[|2\rangle\langle 1|] \rho+\gamma_{2 \rightarrow 1} \mathbb{D}[|1\rangle\langle 2|] \rho$,

with $\mathbb{D}[L]$ denoting the usual Lindblad damping superoperator for jump operator $L$. The resulting $4 \times 4$ Liouvillian $\mathbb{L}$ is block diagonal, where one of the two blocks fully captures the dynamics of density matrices of the form $\rho(t)=p_{1}(t)|1\rangle\left\langle 1\left|+p_{2}(t)\right| 2\right\rangle\langle 2|$, where the probabilities $p_{1,2}$ obey the rate equation (4). This model can be extended and made more realistic by considering subsets of pure states that make up the two metastable states $\rho_{1}$ and $\rho_{2}$, which are likely to be mixed states rather than pure states.

We thank Iacopo Carusotto, Cristiano Ciuti, and Mark Dykman for helpful discussions. This work was supported by the Army Research Office through Grant No. W911NF15-1-0397 and the National Science Foundation through Grants No. DMR-0953475, No. PHY-1055993, and No. PHY-4907160. N. M. S. was supported financially by a NDSEG fellowship.

[1] M.Z. Hasan and C. L. Kane, Colloquium: Topological Insulators, Rev. Mod. Phys. 82, 3045 (2010).

[2] M. W. Zwierlein, J. R. Abo-Shaeer, A. Schirotzek, C. H. Schunck, and W. Ketterle, Vortices and Superfluidity in a
Strongly Interacting Fermi Gas, Nature (London) 435, 1047 (2005).

[3] E. M. Kessler, G. Giedke, A. Imamoglu, S. F. Yelin, M. D. Lukin, and J. I. Cirac, Dissipative Phase Transition in a Central Spin System, Phys. Rev. A 86, 012116 (2012).

[4] H.-P. Breuer and F. Petruccione, The Theory of Open Quantum Systems (Oxford University Press, New York, 2007).

[5] A. A. Houck, H. E. Türeci, and J. Koch, On-Chip Quantum Simulation with Superconducting Circuits, Nat. Phys. 8, 292 (2012).

[6] M. J. Hartmann, Quantum Simulation with Interacting Photons, J. Opt. 18, 104005 (2016).

[7] K. M. Birnbaum, A. Boca, R. Miller, A. D. Boozer, T. E. Northup, and H. J. Kimble, Photon Blockade in an Optical Cavity with One Trapped Atom, Nature (London) 436, 87 (2005).

[8] C. Lang et al., Observation of Resonant Photon Blockade at Microwave Frequencies Using Correlation Function Measurements, Phys. Rev. Lett. 106, 243601 (2011).

[9] A. J. Hoffman, S. J. Srinivasan, S. Schmidt, L. Spietz, J. Aumentado, H. E. Türeci, and A. A. Houck, Dispersive Photon Blockade in a Superconducting Circuit, Phys. Rev. Lett. 107, 053602 (2011).

[10] D. L. Underwood, W. E. Shanks, J. Koch, and A. A. Houck, Low-Disorder Microwave Cavity Lattices for Quantum Simulation with Photons, Phys. Rev. A 86, 023837 (2012).

[11] T. Jacqmin, I. Carusotto, I. Sagnes, M. Abbarchi, D. D. Solnyshkov, G. Malpuech, E. Galopin, A. Lemaître, J. Bloch, and A. Amo, Direct Observation of Dirac Cones and a Flatband in a Honeycomb Lattice for Polaritons, Phys. Rev. Lett. 112, 116402 (2014).

[12] A. D. Greentree, C. Tahan, J. H. Cole, and L. Hollenberg, Quantum Phase Transitions of Light, Nat. Phys. 2, 856 (2006).

[13] M. J. Hartmann, F. Brandão, and M. Plenio, Strongly Interacting Polaritons in Coupled Arrays of Cavities, Nat. Phys. 2, 849 (2006).

[14] D. G. Angelakis, M. F. Santos, and S. Bose, PhotonBlockade-Induced Mott Transitions and XY Spin Models in Coupled Cavity Arrays, Phys. Rev. A 76, 031805 (2007).

[15] J. Cho, D. G. Angelakis, and S. Bose, Fractional Quantum Hall State in Coupled Cavities, Phys. Rev. Lett. 101, 246809 (2008).

[16] A. Petrescu, A. A. Houck, and K. LeHur, Anomalous Hall Effects of Light and Chiral Edge Modes on the Kagome Lattice, Phys. Rev. A 86, 053804 (2012).

[17] B. M. Anderson, R. Ma, C. Owens, D. I. Schuster, and J. Simon, Engineering Topological Many-Body Materials in Microwave Cavity Arrays, Phys. Rev. X 6, 041043 (2016).

[18] J. Raftery, D. Sadri, S. Schmidt, H. E. Türeci, and A. A. Houck, Observation of a Dissipation-Induced Classical to Quantum Transition, Phys. Rev. X 4, 031043 (2014).

[19] P. Roushan et al., Chiral Ground-State Currents of Interacting Photons in a Synthetic Magnetic field, Nat. Phys., doi: (2016).

[20] J. M. Fink, A. Dombi, A. Vukics, A. Wallraff, and P. Domokos, Observation of the Photon-Blockade Breakdown Phase Transition, arXiv:1607.04892.

[21] J. A. Schreier et al., Suppressing Charge Noise Decoherence in Superconducting Charge Qubits, Phys. Rev. B 77, 180502 (2008). 
[22] J. D. Strand, M. Ware, F. Beaudoin, T. A. Ohki, B. R. Johnson, A. Blais, and B. L. T. Plourde, First-Order Sideband Transitions with Flux-Driven Asymmetric Transmon Qubits, Phys. Rev. B 87, 220505(R) (2013).

[23] See Supplemental Material at http://link.aps.org/ supplemental/10.1103/PhysRevX.7.011016 for more details regarding the experiment and mean-field simulations.

[24] U. Naether, F. Quijandría, J. J. García-Ripoll, and D. Zueco, Stationary Discrete Solitons in a Driven Dissipative BoseHubbard Chain, Phys. Rev. A 91, 033823 (2015).

[25] J. Koch, T. M. Yu, J. Gambetta, A. A. Houck, D. I. Schuster, J. Majer, A. Blais, M. H. Devoret, S. Girvin, and R. J. Schoelkopf, Charge-Insensitive Qubit Design Derived from the Cooper Pair Box, Phys. Rev. A 76, 042319 (2007).

[26] M. Biondi, G. Blatter, H. E. Türeci, and S. Schmidt, Nonequilibrium Phase Diagram of the Driven-Dissipative Photonic Lattice, arXiv:1611.00697.

[27] R. Vijay, M. H. Devoret, and I. Siddiqi, Invited Review Article: The Josephson Bifurcation Amplifier, Rev. Sci. Instrum. 80, 111101 (2009).

[28] M. I. Dykman and V. N. Smelyanskiǔ, Quantum Theory of Transitions between Stable States of a Nonlinear Oscillator Interacting with a Medium in a Resonant Field, Sov. Phys. JETP 67, 1769 (1988).

[29] M. Marthaler and M. I. Dykman, Switching via Quantum Activation: A Parametrically Modulated Oscillator, Phys. Rev. A 73, 042108 (2006).

[30] P. D. Drummond and D.F. Walls, Quantum Theory of Optical Bistability. I. Nonlinear Polarisability Model, J. Phys. A 13, 725 (1980).

[31] W. Casteels, F. Storme, A. LeBoite, and C. Ciuti, Power Laws in the Dynamic Hysteresis of Quantum Nonlinear Photonic Resonators, Phys. Rev. A 93, 033824 (2016).
[32] W. H. Zurek, U. Dorner, and P. Zoller, Dynamics of a Quantum Phase Transition, Phys. Rev. Lett. 95, 105701 (2005).

[33] R. M. Wilson, K. W. Mahmud, A. Hu, A. V. Gorshkov, M. Hafezi, and M. Foss-Feig, Collective Phases of Strongly Interacting Cavity Photons, Phys. Rev. A 94, 033801 (2016).

[34] K. Macieszczak, M. Guţă, I. Lesanovsky, and J. P. Garrahan, Towards a Theory of Metastability in Open Quantum Dynamics, Phys. Rev. Lett. 116, 240404 (2016).

[35] C. Eichler, D. Bozyigit, and A. Wallraff, Characterizing Quantum Microwave Radiation and Its Entanglement with Superconducting Qubits Using Linear Detectors, Phys. Rev. A 86, 032106 (2012).

[36] R. Ma, C. Owens, A. LaChapellle, D. I. Schuster, and J. Simon, Hamiltonian Tomography of Photonic Lattices, arXiv:1607.05180.

[37] F. Wulschner et al., Tunable Coupling of Transmission-Line Microwave Resonators Mediated by an rf SQUID, EPJ Quantum Techno. 3, 10 (2016).

[38] J. F. Poyatos, J. I. Cirac, and P. Zoller, Quantum Reservoir Engineering with Laser Cooled Trapped Ions, Phys. Rev. Lett. 77, 4728 (1996).

[39] K. W. Murch, U. Vool, D. Zhou, S. J. Weber, S. M. Girvin, and I. Siddiqi, Cavity-Assisted Quantum Bath Engineering, Phys. Rev. Lett. 109, 183602 (2012).

[40] M. Hafezi, P. Adhikari, and J. M. Taylor, Chemical Potential for Light by Parametric Coupling, Phys. Rev. B 92, 174305 (2015).

[41] A. Potts, G. J. Parker, and P. A. J. Groot, CMOS Compatible Fabrication Methods for Submicron Josephson Junction Qubits, IEE Proc. Sci. Meas. Technol. 148, 225 (2001). 